\title{
The Shape of Covariantly Smeared Sources in Lattice QCD
}

\author{
Georg M. von Hippel, ${ }^{a}$ Benjamin Jäger, ${ }^{a, b}$ Thomas D. Rae ${ }^{a}$ and Hartmut Wittig ${ }^{a, b}$ \\ ${ }^{a}$ PRISMA Cluster of Excellence and Institut für Kernphysik, University of Mainz, 55099 Mainz, \\ Germany \\ ${ }^{b}$ Helmholtz Institute Mainz, University of Mainz, 55099 Mainz, Germany \\ E-mail: hippel@kph.uni-mainz.de, jaeger@kph.uni-mainz.de, \\ thrae@kph.uni-mainz.de, wittig@kph.uni-mainz.de
}

\begin{abstract}
Covariantly smeared sources are commonly used in lattice QCD to enhance the projection onto the ground state. Here we investigate the dependence of their shape on the gauge field background and find that the presence of localized concentrations of magnetic field can lead to strong distortions which reduce the smearing radii achievable by iterative smearing prescriptions. In particular, as $a \rightarrow 0$, iterative procedures like Jacobi smearing require increasingly large iteration counts in order to reach physicallysized smearing radii $r_{\mathrm{sm}} \sim 0.5 \mathrm{fm}$, and the resulting sources are strongly distorted. To bypass this issue, we propose a covariant smearing procedure ("free-form smearing") that allows us to create arbitrarily shaped sources, including in particular Gaussians of arbitrary radius.
\end{abstract}

KEYWORDS: lattice QCD, hadronic wavefunctions, gauge covariance 


\section{Contents}

1 Introduction 1

2 Covariant smearing: a general framework 2

3 Empirical evidence for magnetic distortion 3

$\begin{array}{lll}4 & \text { Covariantly smearing with arbitrary shapes } & 7\end{array}$

5 Discussion and Outlook $\quad 8$

\section{Introduction}

The construction of interpolating operators optimizing the overlap with the desired states in correlation functions is a crucial problem in any effort to extract reliable information from lattice QCD, since contaminations from excited states can constitute a significant source of systematic error. This is particularly important in the baryonic sector, where the signal-to-noise ratio decays rapidly with Euclidean time (cf. e.g. [1-6]). Procedures to isolate the ground and excited states, such as the variational method [7-9], also rely on the ability to construct bases of linearly independent operators. One of the most widely used methods to create operators with improved projection properties is the use of covariant smearing operations such as Gaussian or Jacobi smearing [10-12]. The intuition behind this is that a hadron should be best described by a state created by a spatially extended operator rather than a pointlike one, guided by the principle that the spatial profile of the extended operator resembles the shape of the hadron in question. Although smearing techniques can be combined with information on the expected symmetry properties of hadronic wave functions $[13,14]$, the procedure remains largely heuristic.

Surprisingly enough, the question of which precise shapes these covariantly smeared operators have, does not appear to have been studied in any detail. While it is readily apparent that the application of Gaussian smearing results in a Gaussian shape on a free gauge configuration, the same does not necessarily apply in the presence of gauge fields.

In this paper, we study the general properties of covariant smearing and report on some unexpected discoveries. In section 2, we set up a general formulation of covariant smearing operations, in order to discuss and investigate the dependence of the smeared source on the gauge field background. We find that the existence of localized fluctuations of the chromomagnetic field can distort the shape of the source away from what one would expect from the free-field case. In section 3 we study the effects of such concentrations of field strength, and find them significant, in particular for high iteration counts of Gaussian smearing, where the resulting sources may, in fact, bear no resemblance to a Gaussian at all. 
In particular, this is an at small lattice spacings, where we find that high iteration counts are needed to achieve reasonable smearing radii. To bypass these limitations, in section 4 we introduce a procedure ("free-form smearing") to create covariantly smeared sources of arbitrary shapes, including Gaussians of arbitrary width. We also show that these sources have much improved projection properties in comparison to their field-distorted counterparts. Some remaining issues and open questions are discussed in section 5 .

\section{Covariant smearing: a general framework}

We begin by recapitulating some basic facts about covariant smearing operations in order to set up a general framework in which the shape of covariantly smeared sources can be analysed.

A general covariant smearing operation can be written as

$$
\widetilde{\psi}(x)=\sum_{y} K(x, y) \psi(y)
$$

with a smearing kernel

$$
K(x, y)=\sum_{\mathcal{P} \in P(x, y)} \omega_{\mathcal{P}} U_{\mathcal{P}}
$$

built from paths $\mathcal{P}$ taken from some set $P(x, y)$ of paths connecting $x$ and $y$, where this set might typically be chosen so as to include all paths below a certain length. Representing a path by a sequence of steps along the coordinate axes, $\mathcal{P}=\left(\mu_{\mathcal{P}, 1}, \ldots, \mu_{\mathcal{P}, l_{\mathcal{P}}}\right), 1 \leq\left|\mu_{\mathcal{P}, j}\right| \leq 3$, and using the conventions that $\hat{e}_{-\mu}=-\hat{e}_{\mu}$ and $U_{-\mu}(x)=U_{\mu}^{\dagger}\left(x-\hat{e}_{\mu}\right)$, we denote by

$$
U_{\mathcal{P}} \equiv U_{\mu_{\mathcal{P}, 1}}(x) \cdots U_{\mu_{\mathcal{P}, l_{\mathcal{P}}}}\left(x+\sum_{j=1}^{l_{\mathcal{P}}-1} \hat{e}_{\mu_{\mathcal{P}, j}}\right)
$$

the product of links required to parallel transport a spinor along that path.

For the usual case of a point source, $\psi(y)=\phi_{0} \delta\left(y-y_{0}\right)$ with a normalized SU(3) vector $\phi_{0} \in \mathbb{C}^{3},\left\|\phi_{0}\right\|=1$, we can perform the sum over $y$, in which case the explicit form of the covariant smearing operation reads

$$
\widetilde{\psi}(x)=\sum_{\mathcal{P} \in P\left(x, y_{0}\right)} \omega_{\mathcal{P}} U_{\mathcal{P}} \phi_{0} .
$$

In practice, covariant smearing operations are not implemented as sums over paths, since the number of paths to take into account would generally be unmanageable (e.g. for smearing a point source over a $5^{3}$ volume, i.e. a very small smearing radius of $r \leq 2 a$, there are 14,005 distinct paths of length up to 6 that can contribute). Instead, iterative procedures like Jacobi or Gaussian smearing [10, 11] are generally employed, where the smeared field is derived from the unsmeared one by the repeated application of a derivative or hopping operator $H$ in the form

$$
\widetilde{\psi}=C\left(1+\kappa_{\mathrm{G}} H\right)^{n} \psi .
$$


However, in theory, it is relatively simple to perform the expansion of eqn. (2.5) to obtain the form eqn. (2.4), for example, by using a similar bottom-up algorithm as has been applied to the perturbative expansion of lattice QCD actions [15]; in practice, the number of paths grows exponentially with $n$, so that the enumeration of all paths contributing for practically relevant values of $n$ becomes infeasible.

A useful measure of the spatial extent of a smeared source is given by the smearing radius defined through

$$
r_{\mathrm{sm}}^{2}=\frac{\sum_{x}\left|x-y_{0}\right|^{2}\|\tilde{\psi}(x)\|^{2}}{\sum_{x}\|\tilde{\psi}(x)\|^{2}},
$$

where $y_{0}$ is the position of the source. The norm $\|\tilde{\psi}(x)\|$ of the smeared source at a given spatial point $x$ can thus be seen as determining the weight of the point $x$, and the norm can thus be identified with the general "shape" of the source.

Taking the norm of the smeared source in eqn. (2.4), we find

$$
\begin{aligned}
\|\widetilde{\psi}(x)\|^{2} & =\left\|\sum_{\mathcal{P} \in P\left(x, y_{0}\right)} \omega_{\mathcal{P}} U_{\mathcal{P}} \phi_{0}\right\|^{2} \\
& =\sum_{\mathcal{P} \in P\left(x, y_{0}\right)} \omega_{\mathcal{P}}^{2}+\sum_{\mathcal{P}_{i} \neq \mathcal{P}_{j} \in P(x, 0)} \omega_{\mathcal{P}_{i}} \omega_{\mathcal{P}_{j}} \phi_{0}^{\dagger} U_{\mathcal{P}_{i}}^{\dagger} U_{\mathcal{P}_{j}} \phi_{0} .
\end{aligned}
$$

Since the smearing does not affect the Dirac structure of the source, a full set of sources can be obtained by averaging over the colour components, giving

$$
\|\widetilde{\psi}(x)\|^{2}=\sum_{\mathcal{P}_{i} \in P\left(x, y_{0}\right)} \omega_{i}^{2}+2 \sum_{\substack{\mathcal{P}_{i}, \mathcal{P}_{j} \in P\left(x, y_{0}\right) \\ i<j}} \omega_{\mathcal{P}_{i}} \omega_{\mathcal{P}_{j}} \operatorname{Re} \operatorname{tr}\left[U_{\mathcal{P}_{i}}^{\dagger} U_{\mathcal{P}_{j}}\right] .
$$

The presence of the last term implies that the shape of the source created by the application of the smearing operation will depend on the gauge field background through

$$
\operatorname{Re} \operatorname{tr}\left[U_{\mathcal{P}_{i}}^{\dagger} U_{\mathcal{P}_{j}}\right]=\int_{\Omega_{i j}} \operatorname{Re} \operatorname{tr}\left[B^{2}+\ldots\right]
$$

where $\Omega_{i j}$ is the surface bounded by $P_{i}$ and $P_{j}$. Therefore, we expect that localized regions of strong chromomagnetic fields will affect the shape and, in turn, also the projection properties of a covariantly smeared source.

\section{Empirical evidence for magnetic distortion}

To demonstrate that localized magnetic flux distorts source shapes, we consider first the case in which a highly localized concentration of magnetic field strength has been inserted into an otherwise trivial background by hand. Specifically, we consider the gauge configuration

$$
U_{\mu}(x)=\left\{\begin{array}{l}
u_{0}, \mu=3, x=(0,0,0,0) \\
\mathbb{1}, \text { else }
\end{array}\right.
$$



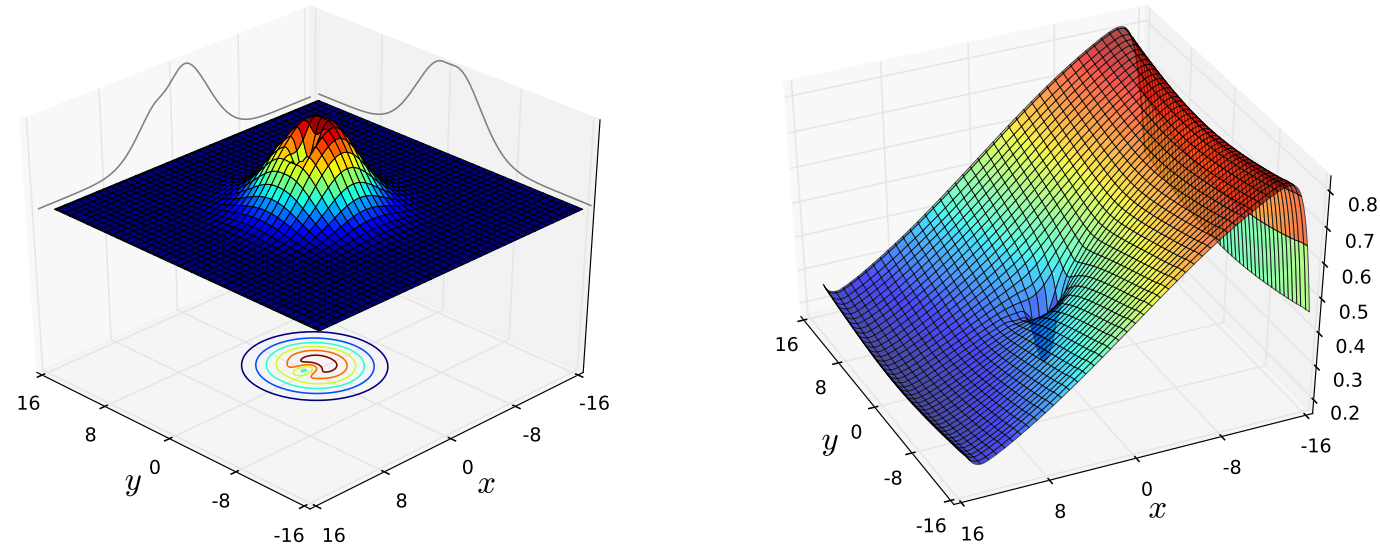

Figure 1. left: the shape of a Gaussian source that has been distorted by an artificially introduced magnetic flux on an otherwise free (unit) configuration; right: the ratio between the Gaussian source distorted by the flux and the same Gaussian source on a free (unit) configuration; it can be seen that besides creating a strong local suppression, the presence of the flux shifts and distorts the source globally.
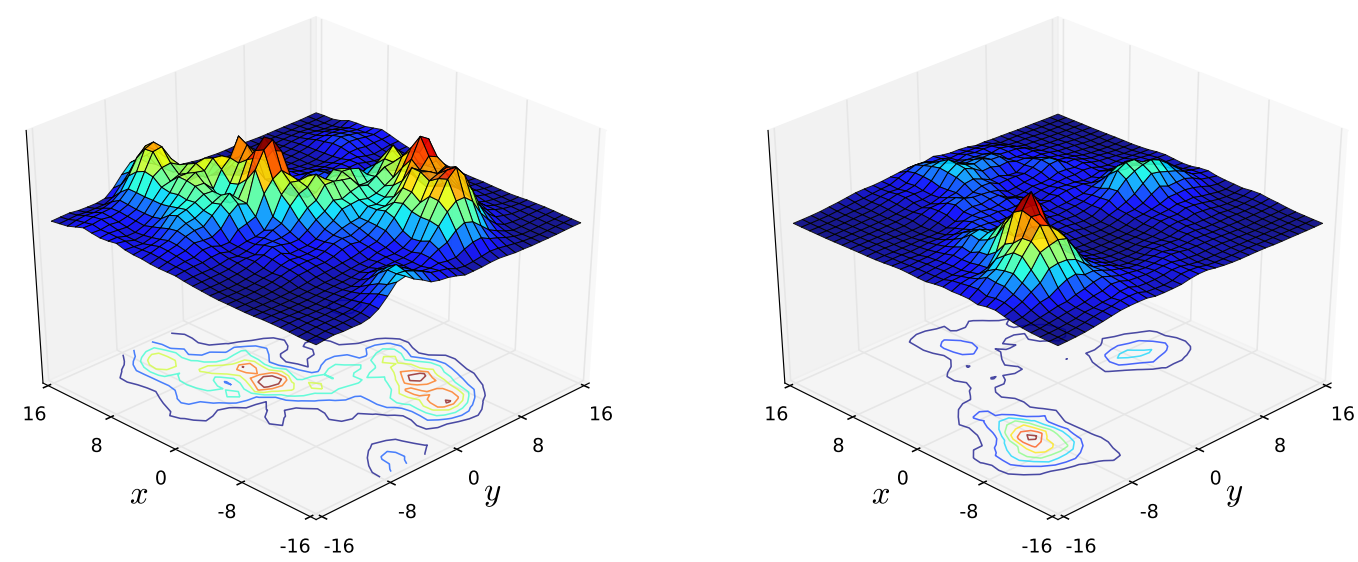

Figure 2. Examples of strongly distorted source shapes encountered by applying eqn. (2.5) with $\kappa_{\mathrm{G}}=2.9, n=640$ to configurations from an $a=0.063 \mathrm{fm}$ ensemble with $N_{\mathrm{f}}=2$ flavours of $\mathrm{O}(a)$-improved Wilson fermions. The corresponding smeared source shape in the free theory would be a broad Gaussian centered on the middle point of this plot.

where $u_{0}$ is chosen far from the identity, and apply Gaussian smearing to the source $\psi(x)=$ $\phi_{0} \delta\left(x-y_{0}\right)$, where $y_{0}=(0, L / 2, L / 2, L / 2)$ and $\phi_{0}$ is a unit vector in Dirac-colour space. For this configuration, the change of the norm of the smeared source compared to the free case is largely a function of the number of contributing paths that pass through the non-unit link.

The resulting source shape can be seen in figure 1. Two features that can be clearly observed are the strong depression of the source at the ends of the non-unit link, as well 

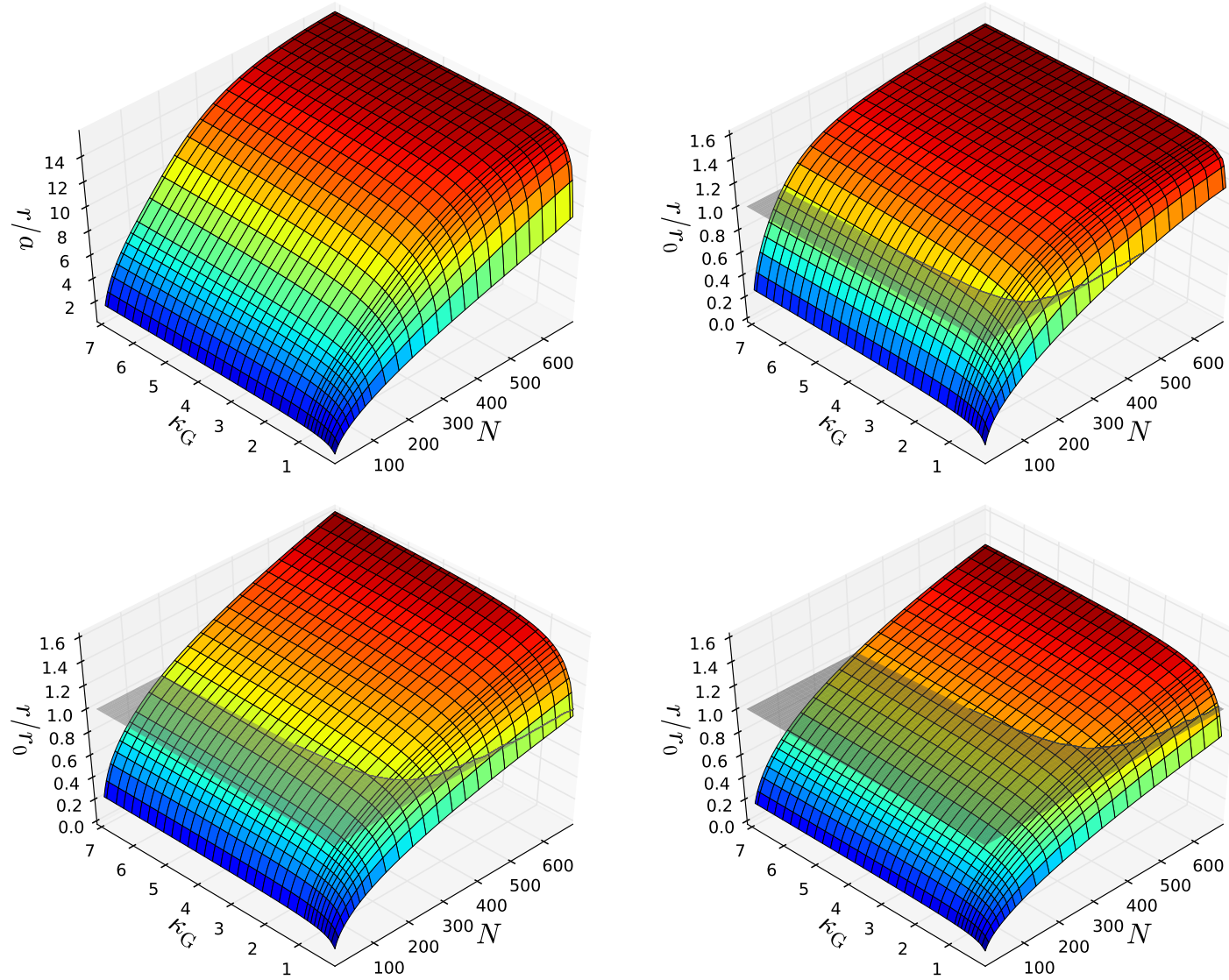

Figure 3. Gaussian "smearscapes" for the free case (top left), an ensemble with $a=0.079 \mathrm{fm}$ (top right), an ensemble with $a=0.063 \mathrm{fm}$ (bottom left), and an ensemble with $a=0.050 \mathrm{fm}$ (bottom right). Shown is the smearing radius $r_{\mathrm{sm}}$ as a function of both $\kappa_{\mathrm{G}}$ and the iteration number $n$. The semitransparent horizontal plane indicates where $r_{\mathrm{sm}}=0.5 \mathrm{fm}$ is achieved in the interacting case. The increasing suppression of the radius as $a \rightarrow 0$ can be clearly seen. For the latter three cases, the smearing radius is plotted in units of $r_{0}$.

as the global distortion of the whole source. Similar distorting effects will also occur in configurations forming part of actual lattice QCD ensembles, as is demonstrated by figure 2 . We see that for large iteration numbers, the distorting effects can be so strong that the resulting shape is very decidedly non-Gaussian.

The presence of these distortions creates problems when attempting to create Gaussian sources with large radii by the iteration of eqn. (2.5), since at large iteration numbers the number of paths contained in the smearing operation grows rapidly, thereby increasing the global influence of the local magnetic flux as more and more paths routed through them start contributing to the source at all points. A consequence of this is that the radii found on actual gauge ensembles will be significantly smaller and grow much more slowly with the iteration number than the corresponding radii in the free theory. This problem can be clearly seen in the "smearscapes" shown in figure 3; for the actual gauge ensembles, 

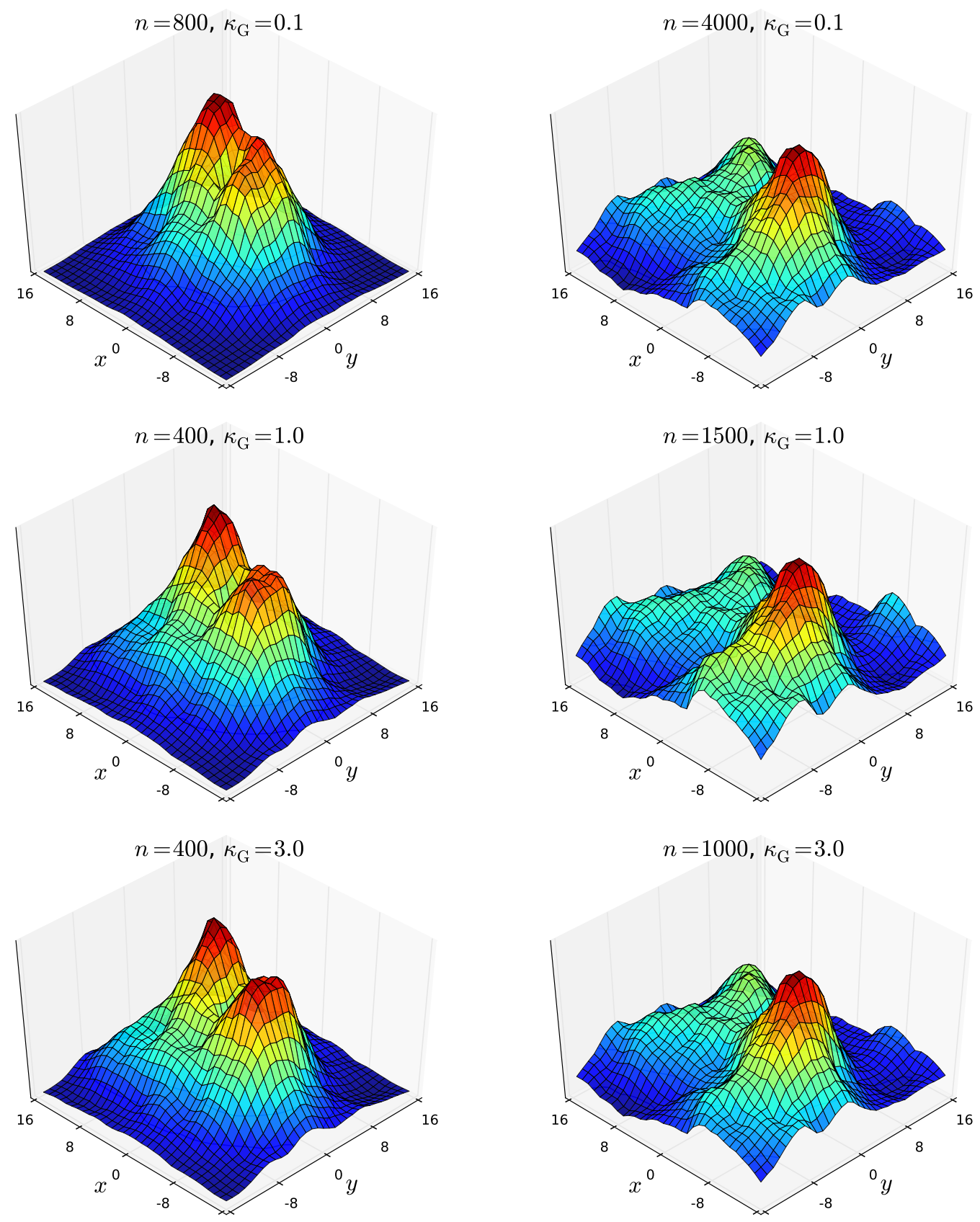

Figure 4. Shapes of covariantly smeared sources on the same $a=0.063 \mathrm{fm}$ gauge configuration for three different parameter sets (from top to bottom: $\kappa_{\mathrm{G}}=0.1,1.0,3.0$ ). On the left, a moderate number of iterations is used, whereas on the right, a large number of iterations has been applied, with the iteration numbers at each value of $\kappa_{\mathrm{G}}$ chosen so as to correspond to approximately the same two smearing radii. It can be seen rather clearly that with increased smearing the source is attracted away from the centre by the influence of the gauge field background, and that for similar smearing radii, similar shapes are observed, regardless of the precise parameters used. 
reaching source radii larger than $r_{\mathrm{sm}} \lesssim 0.5 \mathrm{fm}$, becomes essentially impossible - even if one were willing to pay the required price in terms of iterations, the resulting sources would have surreal shapes (cf. figure 2) bearing absolutely no similarity to a Gaussian on most configurations.

Further evidence that the limits on the achievable smearing radii come from the distortion of the source shapes comes from studying the dependence of the source shape on the parameters $\kappa_{\mathrm{G}}, n$ of the Gaussian smearing. In figure 4, moderately and highly smeared sources for three parameter sets corresponding to approximately the same pair of radii are shown. It can be seen that the same distortion occurs in all three cases.

\section{Covariantly smearing with arbitrary shapes}

In order to overcome some of the limitations that these effects may impose on achievable smearing radii, as well as to avoid the need to gauge-fix an ensemble for introducing nonGaussian source shapes such as hydrogenic wavefunctions, let us return to eqn. (2.4) and consider the possibility of creating an arbitrarily shaped source satisfying $\|\tilde{\psi}(x)\|=\mid f(x-$ $\left.y_{0}\right) \mid\left\|\phi_{0}\right\|$ with an arbitrary function $f$. In a gauge-fixed framework, we might simply use $\tilde{\psi}(x)=f\left(x-y_{0}\right) \phi_{0}$ to this end, which would correspond to setting $K(x, y)=f(x-y)$ in eqn. (2.4). This procedure can be rendered gauge covariant by singling out one particular path $\mathcal{P}_{x y}$ from $y$ to $x$ and defining

$$
K(x, y)=f(x-y) U_{\mathcal{P}_{x y}} .
$$

This would seem to entirely avoid the influence of magnetic fluxes, since there are no different paths that could interfere with each other, and hence the desired relation $\|\tilde{\psi}(x)\|=$ $\left|f\left(x-y_{0}\right)\right|\left\|\phi_{0}\right\|$ holds regardless of the gauge field.

However, this radical solution is not actually practical, since singling out a specific path makes the source much too vulnerable to ultraviolet fluctuations of the individual links, leading to large statistical errors in the correlation functions built from such sources. The same applies if one averages over a moderately-sized set of paths, for example the one created by reflecting a single path along the different coordinate axes.

As an alternative solution, we should therefore consider defining a sufficiently large collection of paths by expanding eqn. (2.5) with a relatively modest iteration count (chosen, for example, as the minimum needed to be able to reach every point on the lattice) into the form of eqn. (2.4), and merely replacing the weights $\omega_{\mathcal{P}}$ so as to reshape the source to obey $\langle\|\tilde{\psi}(x)\|\rangle=\left|f\left(x-y_{0}\right)\right|\left\|\phi_{0}\right\|$ in the gauge average. Unfortunately, this solution is rendered non-viable by the prohibitive cost of enumerating all paths for the iteration numbers required on typical lattices. We can, however, bypass the need to actually enumerate all paths by applying the following procedure:

- calculate $\psi^{\prime}(x)=\left[\left(1+\kappa_{\mathrm{G}} H\right)^{n}\right]_{x, y} \delta\left(y-y_{0}\right) \phi_{0}$, with $n$ chosen large enough to touch all points by at least one path,

- determine $N(x)=\left\langle\left\|\psi^{\prime}(x)\right\|\right\rangle$, 
- define $\tilde{\psi}(x)=\psi^{\prime}(x) f\left(x-y_{0}\right) / N(x)$,

in order to create an arbitrarily-shaped source. We shall call this procedure "free-form smearing". 1

In particular, free-form smearing can be used to create Gaussian source shapes of arbitrary radius without encountering significant distortions of the source shape, thus bypassing the limitations on the source radius found in the preceding section. It can also be used to create anisotropic sources similar to those considered to study moving hadrons in [16], or to build a basis of operators for the application of methods to extract excited state spectra $[7-9,17-20]$. In the latter case, the ability of free-form smearing to create shapes other than Gaussian is particularly useful, since it enables the use of ansätze with nodes, such as hydrogenic wavefunctions, without requiring us to fix the gauge (as had to be done for that purpose in, e.g., [21]).

A comparison of the ideal source shape $f\left(x-y_{0}\right)$ and the result of applying free-form smearing to a given gauge configuration can be seen in figure 5 for the case of both a Gaussian and an arbitrarily chosen source shape $f(z)=\left(1-\sin \left(\pi r^{2} / 50 a^{2}\right)\right) \exp \left(-r^{2} / 72 a^{2}\right)$ displaying nodes. It can be seen that in spite of the fluctuations due to the gauge fields, the free-form smeared shape is well-reproduced even on an individual gauge configuration.

To compare the projection properties of free-form smeared Gaussians of various radii with those of standard Gaussian smearing, we have computed the corresponding hadron correlators on a range of ensembles ${ }^{2}$; the effective masses for both the pion and nucleon channels on the various ensembles can be seen in figures 6 to 8 . It is evident that free-form smearing allows us to use large radii, thus strongly improving the overlap with the ground state. The effect is particularly dramatic in the nucleon channel. As a consequence, a much earlier onset of the plateau region is observed, which significantly improves the statistical precision of the fitted plateau value for the mass, while maintaining a good handle on the contaminations from excited states. This is of particular importance in the nucleon sector, where the rapid growth of the noise-to-signal ratio imposes a limit on how late one can begin a plateau fit with reasonable statistical errors, whereas the systematic errors incurred from excited-state contributions limit how early a reasonably safe plateau can be found. Suppressing the unwanted excited-state contributions thus allows for earlier, much more statistically accurate plateau fits.

\section{Discussion and Outlook}

In lattice simulations, the use interpolating operators with good projection properties to the ground state is crucial, in particular in the baryon sector, where the signal-to-noise ratio deteriorates rapidly with the Euclidean time separation between source and sink, making an early onset of the ground state plateau region highly desirable. Covariant smearing

\footnotetext{
${ }^{1}$ An obvious alternative would be to enforce $\left\langle\|\tilde{\psi}(x)\|^{2}\right\rangle=\left|f\left(x-y_{0}\right)\right|^{2}\left\|\phi_{0}\right\|^{2}$ by defining $N(x)=$ $\sqrt{\left\langle\left\|\psi^{\prime}(x)\right\|^{2}\right\rangle}$

${ }^{2}$ The ensembles used were generated within the Coordinated Lattice Simulations (CLS) initiative [22] using the DD-HMC algorithm [23, 24].
} 

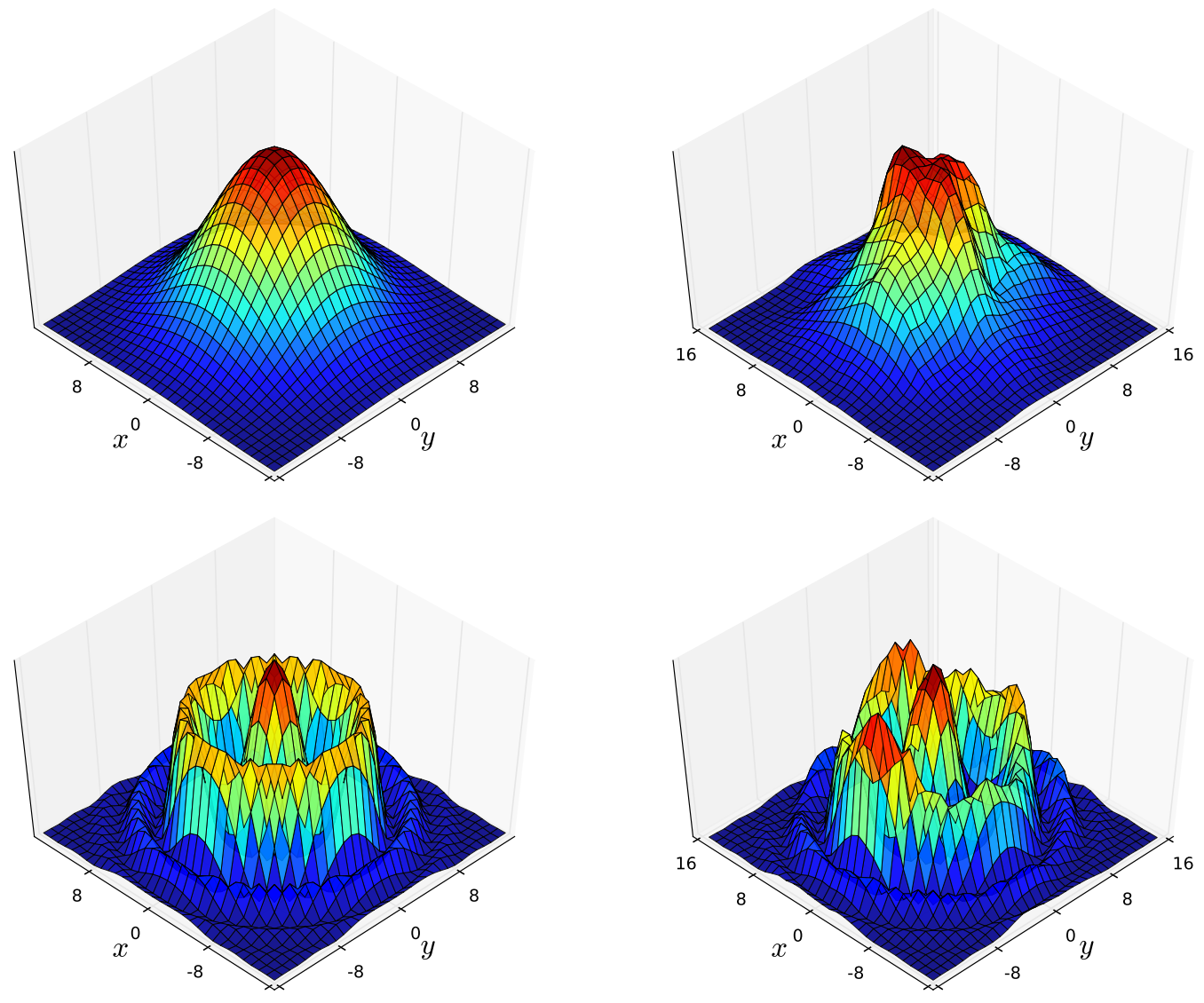

Figure 5. A comparison of the shapes of free-form smeared sources on a unit configuration (left) and a configuration taken from an $a=0.063 \mathrm{fm}$ ensemble (right); shown are an free-form smeared Gaussian source (top) and a source with $f(z)=\left(1-\sin \left(\pi r^{2} / 50 a^{2}\right)\right) \exp \left(-r^{2} / 72 a^{2}\right)$ demonstrating the possibility to free-form smear arbitrary source shapes (bottom).

operations are commonly used to improve the overlap with the ground state in correlation function. Unfortunately, as $a \rightarrow 0$, iterative procedures rapidly require high iteration numbers to realize the smearing radii associated with a good ground-state overlap, and we have seen that the resulting sources bear no resemblance to the Gaussian shapes one would expect.

This strong distortion of the shape of iteratively covariantly smeared sources found at large iteration counts leads to a number of problems: firstly, it becomes increasingly hard to reach large smearing radii, and secondly, the heuristic motivation for smearing is lost: it is not clear why one should expect such strongly distorted sources to provide an improved overlap with the hadronic ground state.

Here, we have given an interpretation of the source shape distortion in terms of interference between paths winding around regions of large chromomagnetic flux on a given configuration in a manner reminiscent of a non-abelian analogue of the Aharonov-Bohm effect, cf. eqn. (2.10). 

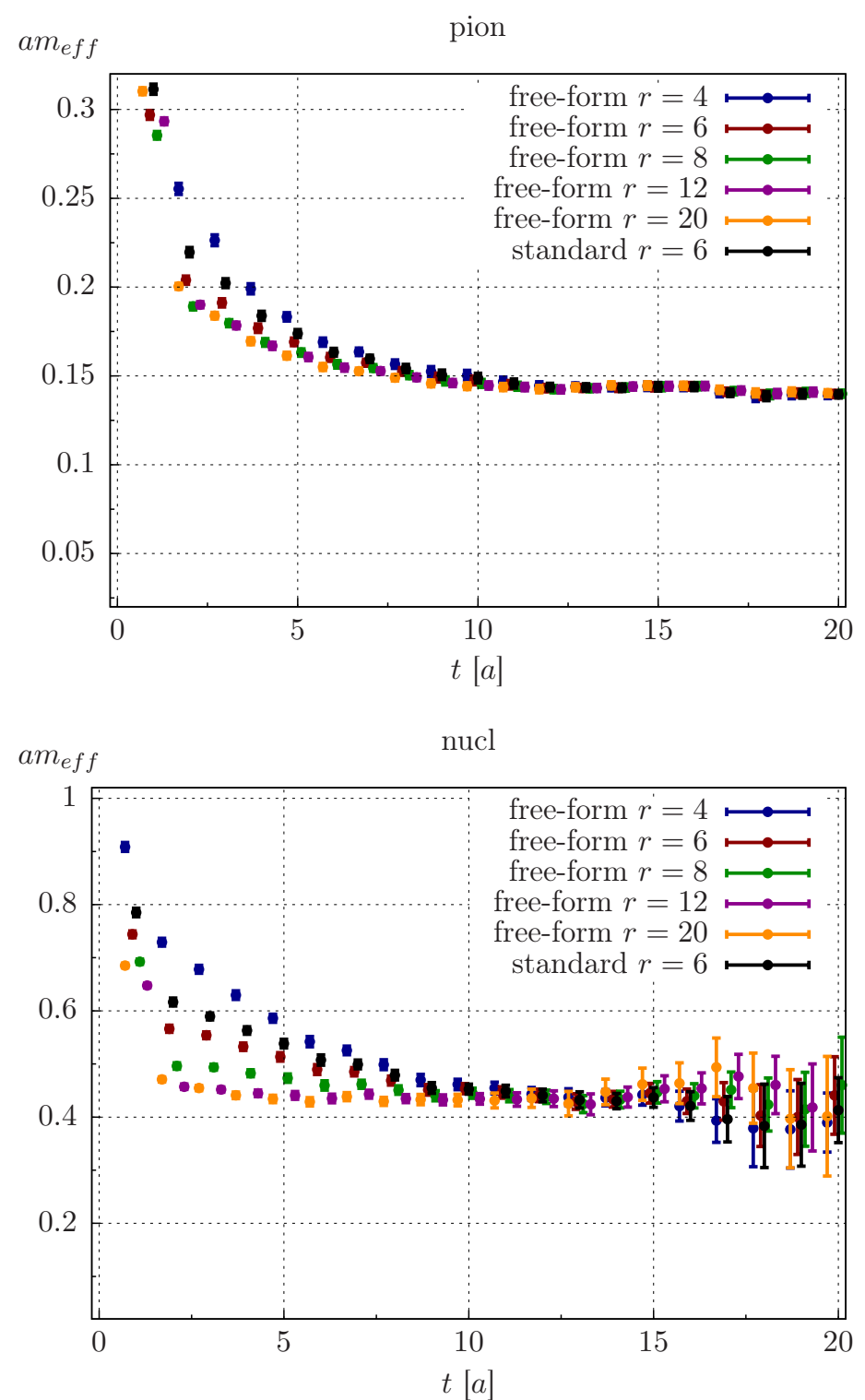

Figure 6. Effective mass plots using free-form smeared Gaussian sources of varying radius for the pion (top) and the nucleon (bottom) on an $a=0.063 \mathrm{fm}$ ensemble with $N_{\mathrm{f}}=2$ flavours of $\mathrm{O}(a)$-improved Wilson fermions $\left(m_{\pi} \approx 450 \mathrm{MeV}, N_{\mathrm{cfg}}=168\right)$. Also shown for comparison is the corresponding result for the Gaussian smearing used to build the set of paths employed; note that the free-form smeared source of the same radius $(r=6 a \approx 0.38 \mathrm{fm})$ gives a slightly faster approach to the plateau. At very large smearing radii it can be seen that the procedure saturates in the sense that no further improvement is seen by going from $r=12 a \approx 0.76 \mathrm{fm}$ to $r=20 a \approx 1.3 \mathrm{fm}$.

To bypass the resulting limitations on the achievable smearing radii, and to enable the use of covariantly smeared sources of arbitrary shape, we propose a new smearing method, "free-form smearing". First tests indicate that free-form smeared sources can reliably reach larger smearing radii and yield better overlap with the ground state than can be achieved 

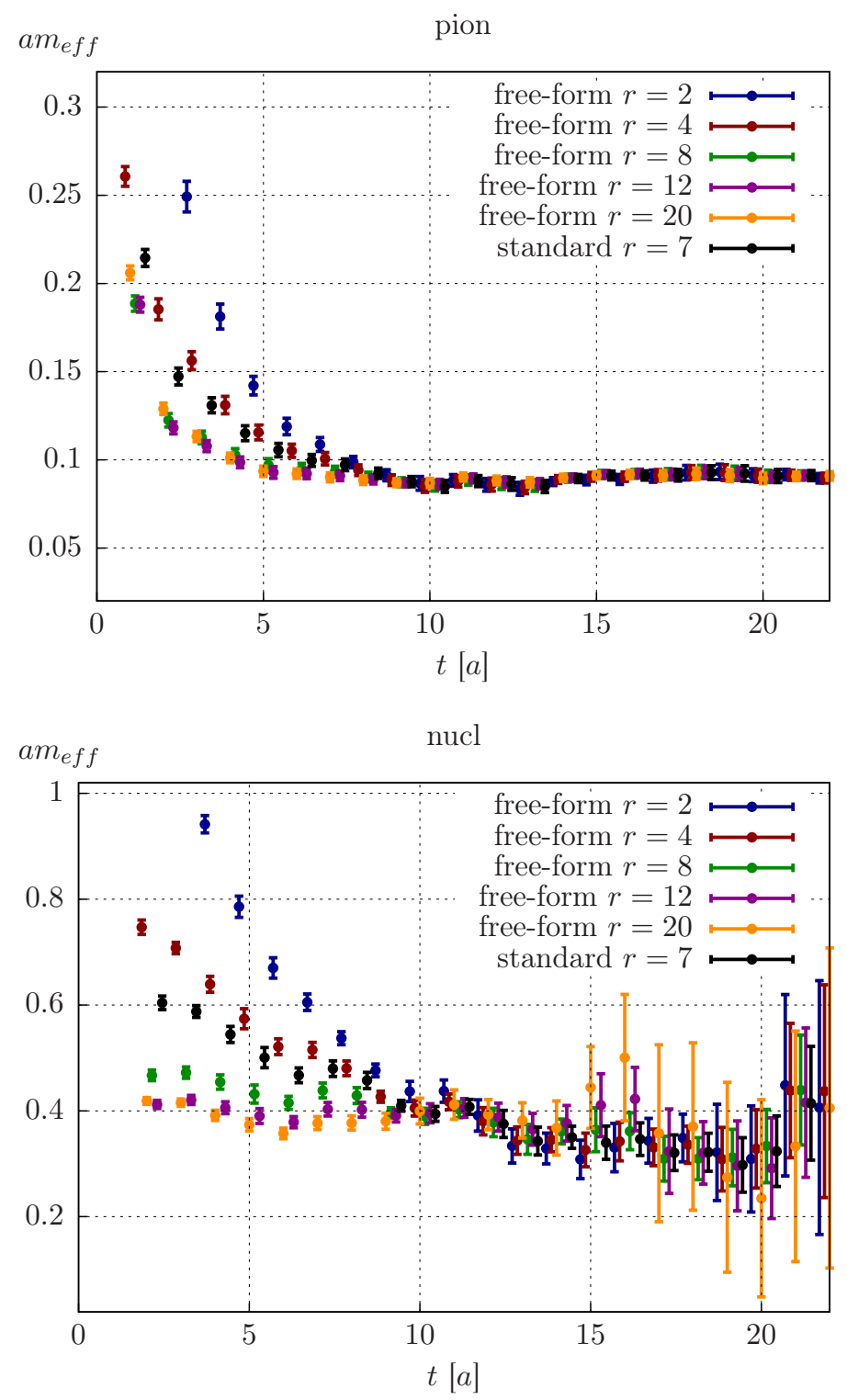

Figure 7. Effective mass plots using free-form smeared Gaussian sources of varying radius for the pion (top) and the nucleon (bottom) on an $a=0.063 \mathrm{fm}$ ensemble with $N_{\mathrm{f}}=2$ flavours of $\mathrm{O}(a)$-improved Wilson fermions $\left(m_{\pi} \approx 280 \mathrm{MeV}, N_{\mathrm{cfg}}=50\right)$. Also shown for comparison is the corresponding result for the Gaussian smearing used to build the set of paths employed. Compared to figure 6 , this ensemble has a lighter pion, and we observe a quicker growth in the statistical errors at large euclidean times; this is particularly true for larger smearing radii, where, however, the earlier onset of the plateau behaviour more than compensates any resulting loss of statistical accuracy.

with the more conventional methods of covariant smearing.

It might be expected that free-form smearing, besides improving the onset of the plateau region for baryons, will be particularly useful for applications in quarkonium spec- 

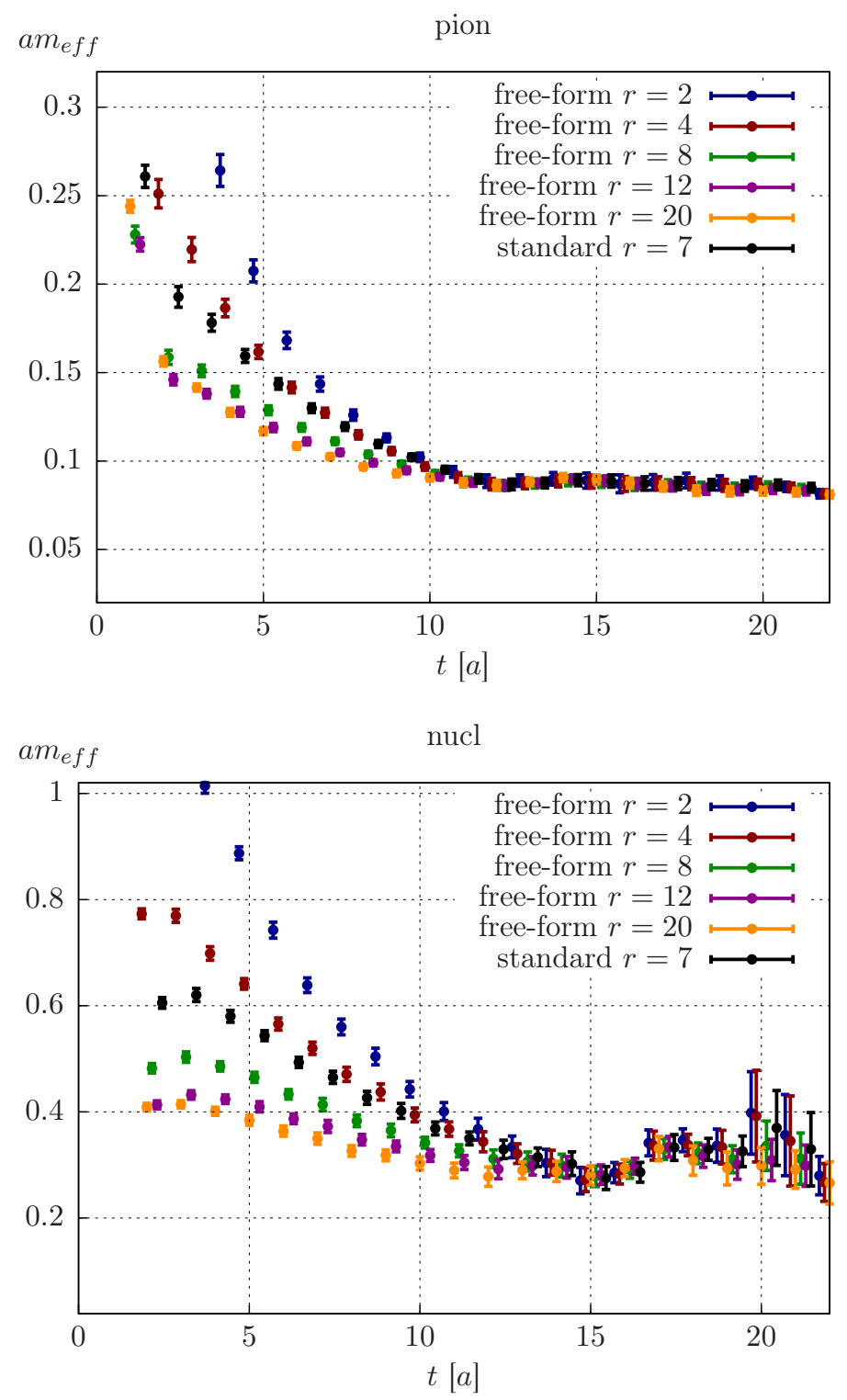

Figure 8. Effective mass plots using free-form smeared Gaussian sources of varying radius for the pion (top) and the nucleon (bottom) on an $a=0.050 \mathrm{fm}$ ensemble with $N_{\mathrm{f}}=2$ flavours of $\mathrm{O}(a)$-improved Wilson fermions $\left(m_{\pi} \approx 320 \mathrm{MeV}, N_{\mathrm{cfg}}=50\right)$. Also shown for comparison is the corresponding result for the Gaussian smearing used to build the set of paths employed. This ensemble has a similar pion mass as the one in figure 6 , but a finer lattice spacing; the efficiency of free-form smearing appears to be only mildly affected by the change in $a$.

troscopy, where the ability to create arbitrary source shapes (such as hydrogenic wavefunctions) is important. A notable limitation of the free-form smearing method is that in its current form it only applies to smearing at the source, precluding its use in the variational method, where the same operators are needed at both source and sink. In order to apply 
free-form smearing at the sink, the rescaling function $N(x)$ would have to be computed with the original point source put at each point on the sink timeslice, leading to a runtime that scales with $V^{2}$. However, a number of methods [18-20] capable of accessing excited state information from a vector of correlation functions exist, for which free-form smearing would appear to be highly suitable.

As a first practical application, we intend to apply free-form smearing to the determination of the lattice spacing from the $\Omega$ mass, which we had previously performed in [25]; the rapid approach to the plateau found for free-form smeared baryonic correlation functions leads us to expect that a significant reduction of both statistical and systematic errors should become possible with free-form smearing in this context.

\section{Acknowledgments}

We acknowledge useful discussions with Rainer Sommer and Hubert Simma.

Our calculations were performed on the "Wilson" HPC Cluster at the Institute for Nuclear Physics, University of Mainz. We thank Dalibor Djukanovic and Christian Seiwerth for technical support. We are grateful for computer time allocated to project HMZ21 on the BG/Q "JUQUEEN" computer at NIC, Jülich. This work was granted access to the HPC resources of the Gauss Center for Supercomputing at Forschungzentrum Jülich, Germany, made available within the Distributed European Computing Initiative by the PRACE2IP, receiving funding from the European Community's Seventh Framework Programme (FP7/2007-2013) under grant agreement RI-283493. This work was supported by the DFG via SFB 1044 and grant HA 4470/3-1.

The $3 \mathrm{~d}$ plots shown in this paper were generated using matplotlib [26].

We are grateful to our colleagues within the CLS initiative for sharing ensembles.

\section{References}

[1] S. Capitani, B. Knippschild, M. Della Morte, and H. Wittig, Systematic errors in extracting nucleon properties from lattice QCD, PoS LATTICE2010 (2010) 147, [1011.1358].

[2] S. Dinter, C. Alexandrou, M. Constantinou, V. Drach, K. Jansen, et al., Precision Study of Excited State Effects in Nucleon Matrix Elements, Phys.Lett. B704 (2011) 89-93, [1108.1076].

[3] C. Alexandrou, S. Dinter, V. Drach, K. Hadjiyiannakou, K. Jansen, et al., A Stochastic Method for Computing Hadronic Matrix Elements, 1302.2608.

[4] J. Green, J. Negele, A. Pochinsky, S. Krieg, and S. Syritsyn, Excited state contamination in nucleon structure calculations, PoS LATTICE2011 (2011) 157, [1111.0255].

[5] J. Green, M. Engelhardt, S. Krieg, J. Negele, A. Pochinsky, et al., Nucleon Structure from Lattice QCD Using a Nearly Physical Pion Mass, 1209.1687.

[6] S. Capitani, M. Della Morte, G. von Hippel, B. Jäger, A. Jüttner, et al., The nucleon axial charge from lattice QCD with controlled errors, Phys.Rev. D86 (2012) 074502, [1205.0180].

[7] C. Michael and I. Teasdale, Extracting Glueball Masses from Lattice QCD, Nucl.Phys. B215 (1983) 433. 
[8] M. Lüscher and U. Wolff, How to calculate the elastic scattering matrix in two-dimensional quantum field theories by numerical simulation, Nucl.Phys. B339 (1990) 222-252.

[9] ALPHA Collaboration, B. Blossier, M. Della Morte, G. von Hippel, T. Mendes, and $\mathrm{R}$. Sommer, On the generalized eigenvalue method for energies and matrix elements in lattice field theory, JHEP 0904 (2009) 094, [0902.1265].

[10] S. Güsken, U. Löw, K. H. Mütter, R. Sommer, A. Patel, et al., Nonsinglet Axial Vector Couplings of the Baryon Octet in Lattice QCD, Phys.Lett. B227 (1989) 266.

[11] C. Alexandrou, F. Jegerlehner, S. Güsken, K. Schilling, and R. Sommer, B meson properties from lattice QCD, Phys.Lett. B256 (1991) 60-67.

[12] UKQCD Collaboration, C. Allton et al., Gauge invariant smearing and matrix correlators using Wilson fermions at Beta = 6.2, Phys.Rev. D47 (1993) 5128-5137, [hep-lat/9303009].

[13] S. Basak, R. Edwards, G. Fleming, U. Heller, C. Morningstar, et al., Group-theoretical construction of extended baryon operators in lattice QCD, Phys.Rev. D72 (2005) 094506, [hep-lat/0506029].

[14] Lattice Hadron Physics Collaboration (LHPC) Collaboration, S. Basak et al., Clebsch-Gordan construction of lattice interpolating fields for excited baryons, Phys.Rev. D72 (2005) 074501, [hep-lat/0508018].

[15] A. Hart, G. M. von Hippel, R. R. Horgan, and E. H. Müller, Automated generation of lattice QCD Feynman rules, Comput. Phys. Commun. 180 (2009) 2698-2716, [0904.0375].

[16] M. Della Morte, B. Jäger, T. Rae, and H. Wittig, Improved interpolating fields for hadrons at non-zero momentum, Eur.Phys.J. A48 (2012) 139, [1208.0189].

[17] J. J. Dudek, R. G. Edwards, N. Mathur, and D. G. Richards, Charmonium excited state spectrum in lattice QCD, Phys.Rev. D77 (2008) 034501, [0707.4162].

[18] G. M. von Hippel, R. Lewis, and R. G. Petry, Evolutionary Fitting Methods for the Extraction of Mass Spectra in Lattice Field Theory, Comput.Phys.Commun. 178 (2008) 713-723, [0707.2788].

[19] K. Hornbostel, G. Lepage, C. Davies, R. Dowdall, H. Na, et al., Fast Fits for Lattice QCD Correlators, Phys.Rev. D85 (2012) 031504, [1111.1363].

[20] G. T. Fleming, S. D. Cohen, H.-W. Lin, and V. Pereyra, Excited-State Effective Masses in Lattice QCD, Phys.Rev. D80 (2009) 074506, [0903.2314].

[21] HPQCD Collaboration, R. Dowdall et al., The Upsilon spectrum and the determination of the lattice spacing from lattice QCD including charm quarks in the sea, Phys.Rev. D85 (2012) 054509, [1110.6887].

[22] Coordinated Lattice Simulations (CLS). https://twiki.cern.ch/twiki/bin/view/CLS/WebIntro.

[23] M. Lüscher, Schwarz-preconditioned HMC algorithm for two-flavour lattice QCD, Comput.Phys.Commun. 165 (2005) 199-220, [hep-lat/0409106].

[24] M. Lüscher, "DD-HMC algorithm for two-flavour lattice QCD." http://luscher. web. cern. ch/luscher/DD-HMC/index.html.

[25] S. Capitani, M. Della Morte, G. von Hippel, B. Knippschild, and H. Wittig, Scale setting via the $\Omega$ baryon mass, PoS LATTICE2011 (2011) 145, [1110.6365]. 
[26] J. D. Hunter, Matplotlib: A 2d graphics environment, Computing In Science 83 Engineering 9 (2007), no. 3 90-95. 